CERN-TH-2019-070

$\mathrm{CP}^{3}$-Origins-2019-20 DNRF90

\title{
The Photon Spectrum of Asymmetric Dark Stars
}

\author{
Andrea Maselli, ${ }^{1, *}$ Chris Kouvaris,,${ }^{2,3, \dagger}$ and Kostas D. Kokkotas ${ }^{4, \ddagger}$ \\ ${ }^{1}$ Dipartimento di Fisica, Sapienza Universitá di Roma E Sezione INFN Roma1, P.A. Moro 5, 00185, Roma, Italy \\ ${ }^{2} \mathrm{CP}^{3}$-Origins, Centre for Cosmology and Particle Physics Phenomenology \\ University of Southern Denmark, Campusvej 55, 5230 Odense M, Denmark \\ ${ }^{3}$ Theoretical Physics Department, CERN, 1211 Geneva, Switzerland \\ ${ }^{4}$ Theoretical Astrophysics, IAAT, University of Tüebingen, Tüebingen 72076, Germany
}

(Dated: December 8, 2020)

\begin{abstract}
Asymmetric Dark Stars, i.e., compact objects formed from the collapse of asymmetric dark matter could potentially produce a detectable photon flux if dark matter particles self-interact via dark photons that kinetically mix with ordinary photons. The morphology of the emitted spectrum is significantly different and therefore distinguishable from a typical black-body one. Given the above and the fact that asymmetric dark stars can have masses outside the range of neutron stars, the detection of such a spectrum can be considered as a smoking gun signature for the existence of these exotic stars.
\end{abstract}

\section{INTRODUCTION}

Today there is strong evidence about the existence of dark matter (DM) from a variety of different sources and scales, such as rotational curves of individual galaxies, clusters of galaxies such as the bullet cluster [1], and the Cosmic Microwave Background [2]. The so-called Collisionless Cold Dark Matter (CCDM) paradigm is well consistent with the observed large scale structure of the Universe. However, this picture changes at small scales. CCDM suffers from several issues that have currently not been resolved within the CCDM scenario. One of them is the core-cusp problem of dwarf galaxies, which is related to the fact that dwarf galaxies are observed to have flat density profiles in their central regions [3, 4], while cuspy profiles for collisionless DM are predicted by N-body simulations [5]. The latter also predict dwarf galaxies with masses that are too large to have not produced stars inside. However such dwarf galaxies have not been observed yet [6], leading to the so called too-bigto-fail problem. Furthermore a diversity problem does exist: galaxies with the same maximum velocity dispersion can differ significantly in the inner core $[7,8]$, in contrast with the results obtained from N-body CCDM simulations. The resolution of such problems can be attributed to different factors: e.g. the inclusion of the baryonic feedback could potentially resolve the core-cusp problem, while statistical deviations from mean values could explain the too-big-to-fail problem. However, an attractive alternative solution to the CCDM inconsistencies is the existence of DM self-interactions (see [9] and reference therein for a review). Self-interactions of the order of $1 \mathrm{~cm}^{2} / \mathrm{g}$ are sufficient, for example, to disperse DM particles and flatten the density profile in dwarf galaxies.

\footnotetext{
* andrea.maselli@roma1.infn.it

$\dagger$ kouvaris@cp3.sdu.dk

‡ kostas.kokkotas@uni-tuebingen.de
}

Moreover, DM self-interactions could resolve another potential problem related to supermassive black holes at high redshifts. Present models of stellar evolution do not seem able to explain how these objects can grow to their current mass within the age of the Universe, if they start as typical stellar remnants. However, collapsing DM can provide the seeds for such supermassive black holes [10].

Given the above, if DM possesses sufficiently strong self-interactions and is of asymmetric nature, it could potentially form compact objects. Asymmetric DM is a viable alternative to the thermally produced DM scenario. An asymmetry in the population between DM particles and antiparticles in the early Universe can lead to the depletion of the antiparticles via annihilations, resulting to the survival of the component in excess. Once annihilations deplete the antiparticle population, no further annihilations can take place simply because DM consists only of particles and not antiparticles. Therefore in such a case, collapsing DM with the appropriate selfinteractions needed to evacuate efficiently the energy, will result in the formation of neutron star-like dark matter objects. The possibility of asymmetric DM forming "dark stars" was first explored in the context of fermionic [11] and bosonic DM [12] where star profiles and basic properties were determined. In addition, the possibility of forming admixed DM-bayonic stars [13, 14] or asymmetric DM cores inside neutron stars (NS) $[15,16]$ also exists. In standard cosmological scenarios, the formation of asymmetric dark stars requires an efficient energy evacuation mechanism. Such a mechanism was described and studied in [17], in which asymmetric DM is assumed to feature self-interactions mediated by a dark photon.Collapsing DM can evacuate energy and shrink further via dark Bremsstrahlung. In this paper was also shown that the collapsing DM cloud can fragment and eventually form dark stars of different masses (depending on the DM and mediator masses and the dark photon coupling).

Asymmetric dark stars could in principle be detected 
via gravitational waves produced from mergers of such objects. The signal can be distinguished from similar mass black hole binaries [18-23] or NS ones [24], exploiting the effect of tidal interactions during the orbital evolution.

Although it seems that it will be difficult to detect dark stars without gravitational wave observations, in this paper here we investigate another possibility given by the emission of a photon flux. Our model is simply described by the following Lagrangian:

$$
\begin{aligned}
\mathcal{L}=\bar{X} & \gamma^{\mu} D_{\mu} X-m_{\mathrm{X}} \bar{X} X-\frac{1}{4} F_{\mu \nu}^{\prime} F^{\prime \mu \nu} \\
& +\frac{1}{2} m_{A^{\prime}}^{2} A_{\mu}^{\prime} A^{\prime \mu}+\frac{1}{2} m_{D}^{2} A_{\mu} A^{\mu}+\frac{\kappa}{2} F_{\mu \nu}^{\prime} F^{\mu \nu}+\ldots,
\end{aligned}
$$

where $X$ is the asymmetric DM particle, which couples to a dark photon $A_{\mu}^{\prime}$ of mass $m_{A^{\prime}}$, and possesses a kinetic mixing with the ordinary photon $\kappa$. We assume that the dark photon acquires its mass via an unspecified Higgs mechanism. The details of this mechanism are not important for the purposes of this work. $D_{\mu}$ is the usual covariant derivative $D_{\mu}=\partial_{\mu}-i g A_{\mu}^{\prime}$ ( $g$ being the coupling to the dark photon). We have omitted the Standard Model part of the Lagrangian. Dark/hidden photons mixing with ordinary photons have been studied thoroughly in literature especially in the context of their effect on stars [25-30]. Eq. (1) contains also a mass term for the ordinary photon. Although this is unacceptable in the vacuum, we will discuss later on how such a mass term can arise in the medium. In the absence of the aforementioned mass term in eq. (1), by shifting appropriately the photon field $A_{\mu}$, one can eliminate the kinetic mixing term from the Lagrangian, inducing a suppressed coupling between dark photon and charged Standard Model (SM) particles, while leaving the dark sector intact, i.e., no coupling between $X$ and $A_{\mu}$. However we will argue later that in the context of a dark star, there are ways to generate such a photon mass term. Shifting the photon field will induce a mixed mass term $\kappa m_{D}^{2} A_{\mu}^{\prime} A^{\mu}$ which upon diagonalization of the mass matrix gives

$$
\mathcal{L}=\bar{X} \gamma^{\mu} D_{\mu} X-m_{\mathrm{X}} \bar{X} X-\frac{1}{4} F_{\Phi \mu \nu} F_{\Phi}^{\mu \nu}-\frac{1}{4} F_{\Phi^{\prime} \mu \nu} F_{\Phi^{\prime}}^{\mu \nu},
$$

where $\Phi$ and $\Phi^{\prime}$ are the new diagonal fields which are linear combinations of the old $A$ and $A^{\prime}$. The covariant derivative now reads $D_{\mu}=\partial_{\mu}-i g \Phi_{\mu}^{\prime}-i g \kappa m_{D}^{2} /\left(m_{A^{\prime}}^{2}-\right.$ $\left.m_{D}^{2}\right) \Phi_{\mu}$. To leading order $A_{\mu} \simeq \Phi_{\mu}$ and therefore SM particles couple mainly to $\Phi$. Therefore there is an effective coupling of the DM $X$ to ordinary photons $\sim g \kappa m_{D}^{2} /\left(m_{A^{\prime}}^{2}-m_{D}^{2}\right)$. Under these assumptions, asymmetric dark stars can produce a spectrum of detectable photons via dark Bremsstrahlung.

Although the overall luminosity of such objects is suppressed by a power of $\kappa$, its magnitude could still be significant, and not dramatically smaller than the luminosity of a standard NS. This somewhat unexpected result can be easily understood. Photons can be produced almost anywhere in the bulk of a dark star and, as long as their mean free path is larger than the stellar radius, they can escape. On the contrary, photons produced by NS are exclusively emitted from their surface, as the mean free path is very small compared to the radius. This volume vs surface effect has also another dramatic impact on the observed flux. Photons produced at different radii inside a dark star are redshifted, as they escape, by a different amount due to the gravitational potential. As we show in the next sections, these features lead to a very peculiar photon spectrum, that is completely different than blackbody radiation, and thus represent a unique smoking gun signal for the discovery of asymmetric dark stars.

Throughout the paper we use natural units, in which $c=\hbar=k_{\mathrm{B}}=1$.

\section{DARK STARS STRUCTURE}

In this work we consider fermionic asymmetric dark stars. We summarise here the basic features of the DM equation of state (EoS), referring the reader to [24] (and reference therein) for more details on the stellar models and their macroscopic properties.

The fermionic DM particles of mass $m_{\mathrm{X}}$ feel the repulsive dark photon interaction which due to the dark photon mass $m_{A^{\prime}}$ behaves as a Yukawa potential of the form

$$
V=\frac{\alpha_{\mathrm{X}}}{r} e^{-m_{A^{\prime}}},
$$

with $\alpha_{\mathrm{X}}$ being the dark fine structure constant. The stellar pressure originates from Fermi exclusion principle and from the short-range interaction (3). Pressure and energy density are computed in the mean field approximation [11] as a function of the DM Fermi momentum $x=p_{\mathrm{F}} / m_{\mathrm{X}}$ :

$$
\begin{aligned}
& \rho=m_{\mathrm{X}}^{4}\left[\frac{\xi(x)}{8 \pi^{2}}+\frac{2}{9 \pi^{3}} \alpha_{\mathrm{X}} \frac{m_{\mathrm{X}}^{2}}{m_{A^{\prime}}^{2}} x^{6}\right], \\
& p=m_{\mathrm{X}}^{4}\left[\frac{\chi(x)}{8 \pi^{2}}+\frac{2}{9 \pi^{3}} \alpha_{\mathrm{X}} \frac{m_{\mathrm{X}}^{2}}{m_{A^{\prime}}^{2}} x^{6}\right] .
\end{aligned}
$$

The two functions $\xi$ and $\chi$ encapsulate the effect of the Fermi-repulsion [31] and read:

$$
\begin{aligned}
& \xi(x)=x \sqrt{1+x^{2}}\left(2 x^{2}+1\right)-\ln \left(x+\sqrt{1+x^{2}}\right), \\
& \chi(x)=x \sqrt{1+x^{2}}\left(2 / 3 x^{2}-1\right)+\ln \left(x+\sqrt{1+x^{2}}\right) .
\end{aligned}
$$

At low (high) densities eqns. (4a)-(4b) reduce to a polytropic $\operatorname{EoS} P=K \rho^{\gamma}$ with index $\gamma \simeq 5 / 3(\gamma \simeq 1)$.

To build the star's structure, we solve Einstein's equations for a spherically symmetric, stationary and static spacetime, supplied by the fermionic EoS. We assume the following ansatz for the non-rotating star:

$$
\begin{aligned}
d s^{2} & =g_{\mu \nu} d x^{\mu} d x^{\nu} \\
& =-e^{2 \nu(r)} d t^{2}+e^{2 \lambda(r)} d r^{2}+r^{2} d \Omega^{2},
\end{aligned}
$$


in the Schwarzschild coordinates $x^{\mu}=(t, r, \theta, \phi)$, where $e^{-2 \lambda(r)}=1-2 G m(r) / r$. The latter leads to the relativistic stellar equations:

$$
\begin{aligned}
\frac{d p}{d r} & =-G \frac{(\rho+p)\left[m(r)+4 \pi r^{3} p\right]}{r[r-2 G m(r)]} \\
\frac{d m}{d r} & =4 \pi r^{2} \rho \\
\frac{d \nu}{d r} & =-\frac{1}{p+\rho} \frac{d p}{d r}
\end{aligned}
$$

which we solve with appropriate boundary conditions at the center of the star, up to the surface, where $r=R$, $m(r)=M$ and $p(R) \rightarrow 0$. The mass-radius profiles of all the configurations considered in this paper, are shown in Fig. (1), for different values of the DM mass, $m_{A^{\prime}}=$ $(10 \mathrm{MeV}, 1 \mathrm{MeV})$ and $\alpha_{\mathrm{X}}=\left(10^{-1}, 10^{-2}\right)$.

\section{BREMSSTRAHLUNG EMISSION}

We consider the emission of Bremsstrahlung photons with momentum $k^{\mu}=(\omega, \vec{k})$, produced in a process of two DM fermions labeled as 1 and 2 scattering to states 3 and 4 (see Fig. 2 for the Feynmann diagram of this process). Each state is defined by the 4-momentum $p_{i}^{\mu}=$ $\left(E_{i}, \vec{p}_{i}\right)$, where $i$ runs from 1 to 4 . The density number of emittedphotons per unit time is given by:

$$
\begin{aligned}
\frac{d^{2} N}{d V d t}=d \Pi \gamma & \int d \Pi|M|^{2} f_{1} f_{2}\left(1-f_{3}\right)\left(1-f_{4}\right)(2 \pi)^{4} \\
& \times \delta^{(4)}\left(p_{1}+p_{2}-p_{3}-p_{4}-k\right)
\end{aligned}
$$

where

$d \Pi=\prod_{i=1}^{4} \frac{d^{3} p_{i}}{(2 \pi)^{3} 2 E_{i}}, \quad d \Pi \gamma=\frac{d^{3} k}{(2 \pi)^{3} 2 \omega}, \quad f_{i}=\frac{1}{e^{\frac{E_{i}-\mu}{T}}+1}$,

with $f_{i}$ being the Fermi function, and $\mu$ and $T$ the DM chemical potential and the star's temperature, respectively. For sake of simplicity, we introduce the variable $X_{i}=\frac{E_{i}-\mu}{T}$, and recast the phase space volume element as:

$$
d^{3} p_{i}=p_{i}^{2} d p_{i} d \Omega_{i} \simeq p_{\mathrm{F}} p_{i} d p_{i} d \Omega_{i},
$$

where $d \Omega_{i}$ is the solid angle specified by $\vec{p}_{i}$, and $p_{\mathrm{F}}$ is the dark particle Fermi momentum, such that $E_{\mathrm{F}}=$ $p_{\mathrm{F}}^{2} /\left(2 m_{\mathrm{X}}\right)$.

The latter can be computed from the star's energy density profile $\rho$, obtained by solving the relativistic stellar structure equations, namely

$$
\rho=\frac{g}{(2 \pi)^{3}} \int_{0}^{\infty} E \frac{d^{3} p}{e^{\frac{E-\mu}{T}}+1} \simeq \frac{1}{\pi^{2}} \int_{0}^{p_{\mathrm{F}}} E p^{2} d p
$$

q where $E=\sqrt{p^{2}+m_{\mathrm{X}}^{2}}$, and $g=2$ reflects the two spin states of the $X$. We assume a system of fermions at low temperaturesuch that the system is degenerate i.e., the chemical potential is much larger than the temperature, $\mu \gg T$. Equation (10) allows to compute the Fermi momentum as a function of the radius inside the star, i.e. $p_{\mathrm{F}}(r)=p_{\mathrm{F}}[\rho(r)]$. As an example, in Fig. 3 we show the values of $p_{\mathrm{F}}$ versus the central energy density corresponding to all the stellar configurations displayed in Fig. 1.

Replacing the expressions (8)-(9) into the master equation (7) then yields:

$$
\begin{aligned}
\frac{d^{2} N}{d V d t}= & \frac{d \Pi \gamma}{16(2 \pi)^{8}} p_{\mathrm{F}}^{4} T^{4} \prod_{i=1}^{4} \int d \Omega_{i}|M|^{2} \delta^{(3)}\left(\vec{p}_{1}+\vec{p}_{2}-\vec{p}_{3}-\vec{p}_{4}-\vec{k}\right) \times \\
& \times \frac{1}{T} \int d X_{i} \frac{1}{e^{X_{1}}+1} \frac{1}{e^{X_{2}}+1} \frac{1}{e^{-X_{3}}+1} \frac{1}{e^{-X_{4}}+1} \delta\left(X_{1}+X_{2}-X_{3}-X_{4}-\omega / T\right)
\end{aligned}
$$

where we have made the assumption that particles are nonrelativistic i.e., $E_{i} \simeq m_{\mathrm{X}}{ }^{1}$. Momentum conservation requires that $\vec{q}=\vec{p}_{1}-\vec{p}_{3}$, and therefore $q^{2}=2 p_{\mathrm{F}}^{2}(1-$ $\left.\cos \theta_{13}\right)\left(\theta_{13}\right.$ being the angle between $\vec{p}_{1}$ and $\left.\vec{p}_{3}\right)$. The scattering amplitude can be factorized in terms of the

\footnotetext{
1 The assumption is a posteriori verified. As it can be inferred from Fig. 3, the Fermi energy is always much smaller than $m_{X}$.
}

elastic scattering amplitude [32] as:

$$
\begin{aligned}
|M|^{2} & =c\left|M_{e l}\right|^{2} \frac{1}{m_{\mathrm{X}}^{2} \omega^{2}}\left(\vec{q}^{2}-\frac{(\vec{q} \cdot \vec{k})^{2}}{\omega^{2}}\right)= \\
& =c\left|M_{e l}\right|^{2} \frac{2 p_{\mathrm{F}}^{2}}{m_{\mathrm{X}}^{2} \omega^{2}}\left(1-\cos \theta_{13}\right)\left(1-\cos ^{2} \theta_{q k}\right),
\end{aligned}
$$

where

$$
c=2(g \epsilon)^{2}=8 \pi \alpha_{\mathrm{X}} \epsilon^{2}
$$

$\theta_{q k}$ the angle between $\vec{q}$ and $\vec{k}$, and $g$ the coupling between DM and dark photons $\left(\alpha_{\mathrm{X}}=g^{2} /(4 \pi)\right)$. Here $\epsilon$ 

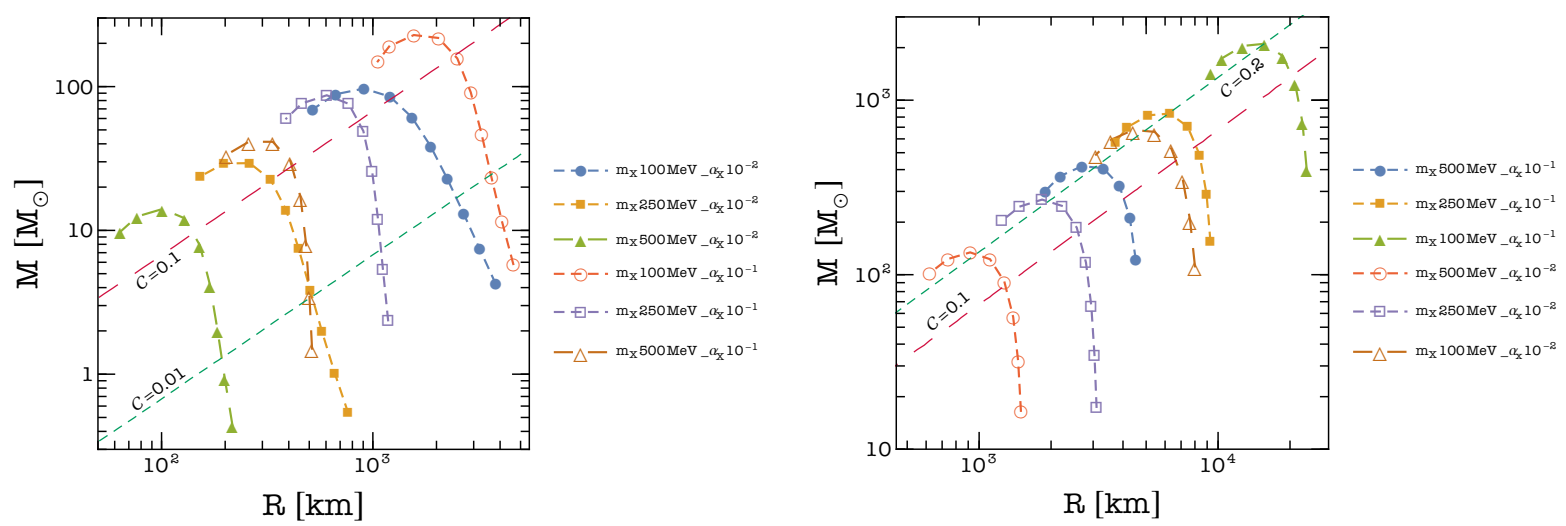

FIG. 1. Mass-radius profiles for the fermionic EoS. The configurations are identified by the dark photon mass $m_{A^{\prime}}=10 \mathrm{MeV}$ (left panel) and $m_{A^{\prime}}=1 \mathrm{MeV}$ (right panel), by the DM mass $m_{\mathrm{X}}=(100,250,500) \mathrm{MeV}$, and by the constant $\alpha_{\mathrm{X}}=\left(10^{-1}, 10^{-2}\right)$. Dashed straight lines identify stellar configurations with constant compactness $\mathcal{C}=M / R$.

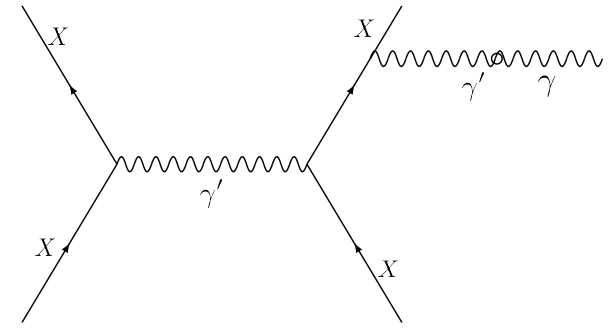

FIG. 2. Feynmann diagram of the Bremsstrahlung process we consider in this paper. Note that the photon can be attached to any DM leg. In the diagonal basis the DM exchange a $\Phi^{\prime}$ and emit a $\Phi$.

specifies the effective mixing between dark the ordinary photons. As mentioned earlier, the kinetic mixing term of eq. (1) can be formally removed by shifting the photon field and therefore in such a case no coupling between DM and photons exists. However, in the case where there is a nonzero photon mass inside the dark star, the aforementioned shift will remove the kinetic mixing term, while introducing a term $\kappa m_{D}^{2} A_{\mu} A^{\prime \mu}$, where $m_{D}$ is the photon mass inside the dark star. Upon diagonalization of the mass matrix, there is an induced DM-photon coupling with strength $-g \kappa m_{D}^{2} /\left(m_{D}^{2}-m_{A^{\prime}}^{2}\right)$. Clearly if the photon remains massless inside the dark star, no coupling between DM and photons exists. There are (at least) three potential scenarios and reasons for a photon to have a nonzero mass inside the dark star: i) DM is in a degenerate state and the vacuum polarization diagram of the photon gets a correction via a loop of DM. This is the usual Debye mass contribution which in the case of $m_{D}>m_{A^{\prime}}$ (and within the hard-dense-loop approximation) takes the simple form $m_{D}=g \kappa \mu / \pi$, where $\mu$ is the chemical potential of DM. In this case $\epsilon=\kappa$. ii) the existence of even small quantities of protons and electrons inside the dark star will also induce a photon Debye mass of the order of $e \mu_{e} / \pi$ where $\mu_{e}$ is the chem-
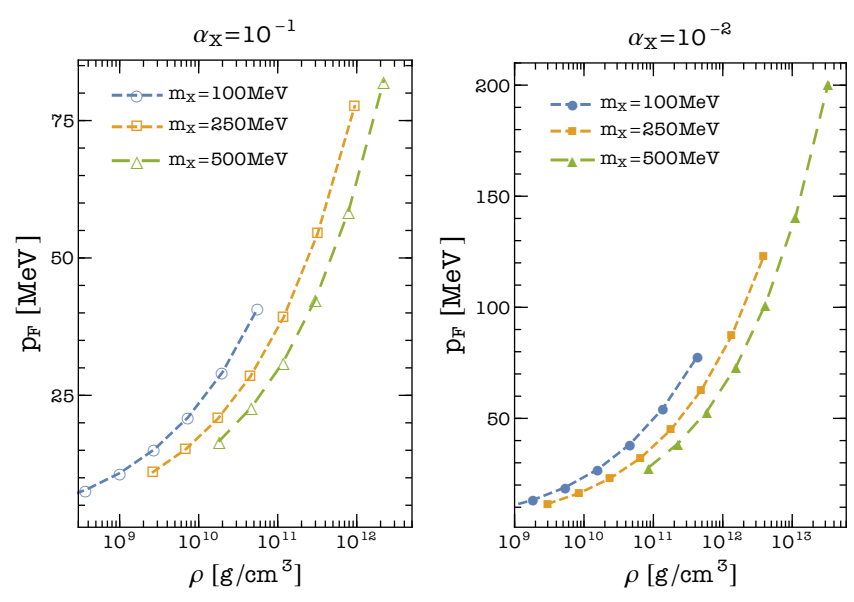

FIG. 3. The DM Fermi momentum as a function of the central energy density for the three different stellar configurations shown in Fig. 1, i.e., $m_{\mathrm{X}}=(100,250,500) \mathrm{MeV}$, and $m_{A^{\prime}}=$ $10 \mathrm{MeV}$. The right end of the lines corresponds to the central energy density of the stellar configuration with the maximum mass for fixed DM and dark photon masses as well as $\alpha_{X}$.

ical potential of electrons ${ }^{2}$. Despite the fact that the amount of electrons inside the star might be negligible to DM, this contribution to the Debye mass comes from the direct coupling of photons to electrons (or protons) and is not suppressed by $\kappa$. iii) Photons might acquire a medium induced mass via a Higgs mechanism. One can envision a Higgs-like scalar field $\phi$ coupled to photons and $\mathrm{DM}$ via an interaction e.g. $\phi \bar{X} X$. The nonzero DM density which translates to a nonzero $\langle\bar{X} X\rangle$ sources in turn a nonzero expectation value for $\phi$, effectively providing a mass for the photon in the DM infested medium (via

\footnotetext{
2 Protons will also be present to maintain overall electric neutrality.
} 
a Higgs-like mechanism), while photons remain massless outside of the star. We leave for future work the precise study of all three scenarios. In this paper we are going to assume that the photon acquire a mass via any of the above mechanisms and we focus on the estimate of the star's luminosity. We should mention at this point that as long as the photon mass is smaller than the tem- perature of the dark star, the kinetic mixing will always induce a Bremsstrahlung process to real photons.

The factor of 2 in eq. (13)comes from the fact that the photon can be emitted by any of the two DM particles that scatter. The amplitude of the elastic process is given by

$$
\left|M_{e l}\right|^{2}=2 g^{4}\left[\frac{s^{2}+t^{2}}{\left(u-m_{A^{\prime}}^{2}\right)^{2}}+\frac{s^{2}+t^{2}}{\left(t-m_{A^{\prime}}^{2}\right)^{2}}+\frac{2 s^{2}}{\left(t-m_{A^{\prime}}^{2}\right)\left(u-m_{A^{\prime}}^{2}\right)}\right]
$$

where $s=\left(p_{1}+p_{2}\right)^{2}, t=\left(p_{1}-p_{3}\right)^{2}$ and $u=\left(p_{1}-p_{4}\right)^{2}$ are the usual Mandelstam variables. To proceed and simplify things we will consider two distinct and orthogonal cases. In the first case we assume that $m_{A^{\prime}}>>p_{F}$, while in the second case we will consider the opposite limit $m_{A^{\prime}}<<$ $p_{F}$. In the first case the amplitude becomes simple based on the fact that $m_{A^{\prime}}^{2}$ will always dominate over $t$ and $u$ in the denominators of eq. (14), and since $s \simeq 4 m_{X}^{2}$, $s>>t$ and $s>>$, the elastic scattering matrix now reads reads:

$$
\left|M_{e l}\right| \simeq \frac{64 g^{4} m_{\mathrm{X}}^{4}}{m_{A^{\prime}}^{4}}
$$

where the numerical prefactor comes from considering all possible diagrams [33]. A symmetry factor of $1 / 2$ has been taken into account based on the fact that the scattering particles are identical. The previous equation is strictly valid when $m_{A^{\prime}}>>p_{\mathrm{F}}$. To obtain this result we have also assumed that the DM particles are nonrelativistic within the star i.e., $m_{X}>>p_{F}$. The rate of photon production is now given by

$$
\frac{d^{2} N}{d V d t}=\frac{c T^{3}}{16(2 \pi)^{11}}\left|M_{e l}\right|^{2} \frac{p_{\mathrm{F}}^{6}}{m_{\mathrm{X}}^{2} \omega^{3}} I_{1} \times I_{2} d^{3} \omega
$$

where we have split the two integrals:

$$
\begin{gathered}
I_{1}=\prod_{i=1}^{4} \int d \Omega_{i} \delta^{(3)}\left(\vec{p}_{1}+\vec{p}_{2}-\vec{p}_{3}-\vec{p}_{4}\right) \\
\quad \times\left(1-\cos \theta_{13}\right)\left(1-\cos ^{2} \theta_{q k}\right) \\
I_{2}=\int \frac{d X_{1}}{e^{X_{1}}+1} \frac{d X_{2}}{e^{X_{2}}+1} \frac{d X_{3}}{e^{-X_{3}}+1} \frac{1}{e^{-X_{1}-X_{2}+X_{3}+\frac{\omega}{T}}+1} .
\end{gathered}
$$

The integration of $X_{i}$ is performed within the interval $\left[-\frac{\mu}{T}, \infty\right]$; however, since $\mu \gg T$, we can assume $\frac{\mu}{T} \rightarrow$ $\infty$, so the integrals can be taken from $[-\infty, \infty]$. In $I_{1}$ we omit $\vec{k}$ from the delta function since the magnitude of the latter should be of the order of the temperature which is much smaller than $\overrightarrow{p_{i}}$ which are practically equal in magnitude to $p_{F}$. In $I_{2}$ we have performed the $X_{4}$ integration using the energy delta function. The integral $I_{1}$ can be computed analytically. Since all $\vec{p}_{i}$ are on the Fermi surface, the only non-trivial phase space comes from back-to-back scattering, i.e. $\vec{p}_{1} \simeq-\vec{p}_{2}$ and $\vec{p}_{3} \simeq-\vec{p}_{4}$. In this case

$$
I_{1}=\frac{1}{p_{F}^{3}} \int d \Omega_{1} d \Omega_{3}\left(1-\cos \theta_{13}\right)\left(1-\cos ^{2} \theta_{q k}\right)=\frac{32 \pi^{2}}{3 p_{\mathrm{F}}^{3}},
$$

and therefore

$$
\frac{d^{2} N}{d V d t}=\frac{c T^{3}}{(2 \pi)^{9}}\left|M_{e l}\right|^{2} \frac{p_{\mathrm{F}}^{3}}{6 m_{\mathrm{X}}^{2} \omega^{3}} I_{2} d^{3} \omega
$$

where $I_{2}=I_{2}(\omega)$.

Combining eqns. (13), (15), and (19), we have

$$
\frac{d^{2} N}{d V d t}=\frac{8}{3} \frac{T^{3}}{\pi^{6}} \frac{m_{\mathrm{X}}^{2}}{m_{A^{\prime}}^{4}} \frac{p_{\mathrm{F}}^{3}}{\omega^{3}} \alpha_{\mathrm{X}}^{3} \epsilon^{2} I_{2} d^{3} \omega
$$

Given that the photon emission is isotropic and $d^{3} \omega=$ $4 \pi \omega^{2} d \omega$, we can rewrite eq. (20) as:

$$
\frac{d^{2} N}{d V d t}=\frac{32}{3 \pi^{5}} T^{3} \frac{m_{\mathrm{X}}^{2}}{m_{A^{\prime}}^{4}} \frac{p_{\mathrm{F}}^{3}}{\omega} \alpha_{\mathrm{X}}^{3} \epsilon^{2} I_{2} d \omega
$$

In the detector reference frame, time interval and energy of emitted photons are redshifted by the source's gravitational field, i.e. $\omega_{\infty}=\sqrt{g_{00}} \omega$ and $d t_{\infty}=d t / \sqrt{g_{00}}$, where the subscript $\infty$ identify redshifted quantities, and $g_{00}$ is the time-time component of the metric tensor inside the star. Note that the latter depends on the radial coordinate $r$, and it matches the analytic Schwarzschild expression at the stellar surface, where $g_{00}=1-2 M / R$. Taking into account the redshift and using eq. (21), we can obtain the flux of photons arriving on an Earth based detector:

$$
\frac{d^{3} N}{d V d S d t_{\infty}}=\frac{8}{3} \frac{\sqrt{g_{00}}}{\pi^{6}} \frac{T^{3}}{d^{2}} \frac{m_{\mathrm{X}}^{2}}{m_{A^{\prime}}^{4}} \frac{p_{\mathrm{F}}^{3}}{\omega_{\infty}} \alpha_{\mathrm{X}}^{3} \epsilon^{2} I_{2} d \omega_{\infty}
$$

where $d S$ is the detector differential area. To get the flux we have divided by a factor of $4 \pi d^{2}$ ( $d$ being the distance between Earth and dark star). The reader should keep in mind that $g_{00}$ is a function of $r$ the distance from the 
center of the star where the photon was emitted. In general, some of the produced photons inside the dark star might not make it out, due to the possibility of converting back to dark photons via dark Compton scattering. This happens when the mean free path of the photon becomes smaller than the distance it must cross within the star in order to get out. We take this absorption effect into account by suppressing the photon flux by an exponential factor of the optical depth of the stellar medium, $\sim e^{-\tau(r)}$, with

$$
\tau(r)=\int_{r}^{R} \sqrt{g_{r r}} n_{\mathrm{X}}(y) \sigma_{\mathrm{c}} d y,
$$

where $n_{\mathrm{X}}$ is the number density of DM particles inside the star, $\sigma_{\mathrm{c}}$ is the photon-DM scattering cross section which is given by the dark Compton one multiplied by a factor of $\epsilon^{2}$, i.e., $\sigma_{\mathrm{c}}=g^{4} \epsilon^{2} /\left(6 \pi m_{\mathrm{X}}^{2}\right)$, and $g_{r r}$ is the $r r$ component of the metric tensor. By including this factor, we can now integrate over the volume of the whole dark star

$$
\frac{d^{3} N}{d S d t_{\infty} d \omega_{\infty}}=\frac{32}{3 \pi^{5}} \frac{T^{3}}{d^{2}} \frac{m_{\mathrm{X}}^{2}}{m_{A^{\prime}}^{4}} \frac{\alpha_{\mathrm{X}}^{3} \epsilon^{2}}{\omega_{\infty}} \int_{0}^{R} \frac{\sqrt{g_{00} g_{r r}}}{e^{\tau(r)}} p_{\mathrm{F}}^{3} I_{2} r^{2} d r .
$$

Finally, the total energy flux arriving at the detector $\mathcal{F}=\frac{d^{2} E}{d S d t_{\infty}}$ is:

$$
\mathcal{F}=\frac{32}{3 \pi^{5}} \frac{T^{4}}{d^{2}} \frac{m_{\mathrm{X}}^{2}}{m_{A^{\prime}}^{4}} \alpha_{\mathrm{X}}^{3} \epsilon^{2} \int_{0}^{\infty} I_{2} d z \int_{0}^{R} \sqrt{g_{00} g_{r r}} \frac{p_{\mathrm{F}}^{3} r^{2}}{e^{\tau}} d r,
$$

where we have introduced the dimensionless variable ${ }^{3}$ $z=\omega_{\infty} / T$. Recall that $p_{F}$ is a function of $r$.

Now we would like to consider the opposite limit $m_{A^{\prime}}<<p_{F}$. This is slightly more complicated because the denominator of each of the three terms in eq. (14) is not simply $\sim m_{A^{\prime}}^{4}$ and $M_{e l}$ becomes angle depended. In this case the factorization of the angle integrals via the $I_{1}$ and $I_{2}$ of Eqs. (17) is not valid anymore. Recall that at the previously examined limit $m_{A^{\prime}}>>p_{F}, M_{e l}$ was independent of angles. At the limit where $m_{A^{\prime}}<<p_{F}$ one can easily show that the second and third term of eq. (14) give contributions of the order of $\sim g^{4} m_{X}^{4} / p_{F}^{4}$, while the first one of $\sim m_{X}^{4} /\left(p_{F}^{2} m_{A^{\prime}}^{2}\right)$ which dominates since in this limit $m_{A^{\prime}}<<p_{F}$. Therefore we will keep only the contribution from this term in the following derivation. Within this approximation the amplitude becomes

$$
\left|M_{e l}\right|^{2}=\left[16 g^{4} \frac{m_{X}^{4}}{m_{A^{\prime}}^{4}}\right] \frac{1}{\left(1+\gamma+\gamma \cos \theta_{13}\right)^{2}},
$$

where $\gamma=2 p_{F}^{2} / m_{A^{\prime}}^{2}$. In the above we have taken into account the symmetry factor of $1 / 2$ due to identical scattering particles. The term within the bracket is the result

\footnotetext{
${ }^{3}$ Note that $I_{2}=I_{2}\left(z / \sqrt{g_{00}}\right)$.
}

for this term in the opposite limit i.e., $m_{A^{\prime}}>>p_{F}$. Here we are interested in light dark photons i.e., $\gamma>>1$. As mentioned earlier, the amplitude becomes dependent on the angle $\theta_{13}$ and is not constant anymore. This means that the photon production rate will still be given by eq. (16), with $\left|M_{e l}\right|^{2}$ being the angle independent piece of the amplitude i.e., the bracket term of eq. (26) and the angle integral $I_{1}$ substituted by an $I_{1}^{\prime}$ which includes the angle dependent part of eq. (26) and reads

$$
I_{1}^{\prime}=\frac{1}{p_{F}^{3}} \int d \Omega_{1} d \Omega_{3} \frac{\left(1-\cos \theta_{13}\right)\left(1-\cos ^{2} \theta_{q k}\right)}{\left(1+\gamma+\gamma \cos \theta_{13}\right)^{2}} .
$$

At the limit where $\gamma<<1$, one recovers $I_{1}^{\prime} \simeq I_{1}$ (see eq. (18)). The angle $\theta_{q k}$ does not depend on the $\theta_{13}$ and we can simplify things by taking into account that $\overline{\cos ^{2} \theta_{q k}}=1 / 3$. Using that, rotating the coordinate system so particle 1 is along the $z$-axis and performing first the trivial integrations over $\Omega_{3}$ and $\phi_{1}$ and then the integration over $\theta_{1}$, gives to leading order in $1 / \gamma$

$$
I_{1}^{\prime}=\frac{16 \pi^{2} m_{A^{\prime}}^{2}}{3 p_{F}^{5}} .
$$

As mentioned above, the new version of eq. (16), is with $\left|M_{e l}\right|^{2}$ being the angle independent the bracket term of eq. (26) and the angle integral $I_{1}$ substituted by $I_{1}^{\prime}$. The photon production rate now reads

$$
\frac{d^{2} N}{d V d t}=\frac{T^{3}}{3 \pi^{6}} \alpha_{X}^{3} \epsilon^{2} \frac{m_{X}^{2}}{m_{A^{\prime}}^{2}} \frac{p_{F}}{\omega^{3}} I_{2} d^{3} \omega
$$

where we have used eq. (13). eq. (29) is the new version of eq. (20) at the limit $m_{A^{\prime}}<<p_{F}$. Performing the trivial angular integral of $d^{3} \omega$ and dividing by $4 \pi d^{2}$ as in eq. (22) we get

$$
\frac{d^{3} N}{d V d S d t_{\infty}}=\frac{T^{3}}{3 \pi^{6} d^{2}} \sqrt{g_{00}} \alpha_{X}^{3} \epsilon^{2} \frac{m_{X}^{2}}{m_{A^{\prime}}^{2}} \frac{p_{F}}{\omega_{\infty}} I_{2} d \omega_{\infty} .
$$

To complete the set of equations, the $m_{A^{\prime}}<<p_{F}$ limit of Eqs. (24) and (25) are given by

$$
\frac{d^{3} N}{d S d t_{\infty} d \omega_{\infty}}=\frac{4}{3 \pi^{5}} \frac{T^{3}}{d^{2}} \frac{m_{\mathrm{X}}^{2}}{m_{A^{\prime}}^{2}} \frac{\alpha_{\mathrm{X}}^{3} \epsilon^{2}}{\omega_{\infty}} \int_{0}^{R} \frac{\sqrt{g_{00} g_{r r}}}{e^{\tau(r)}} p_{\mathrm{F}} I_{2} r^{2} d r
$$

and

$$
\mathcal{F}=\frac{4}{3 \pi^{5}} \frac{T^{4}}{d^{2}} \frac{m_{\mathrm{X}}^{2}}{m_{A^{\prime}}^{2}} \alpha_{\mathrm{X}}^{3} \epsilon^{2} \int_{0}^{\infty} I_{2} d z \int_{0}^{R} \sqrt{g_{00} g_{r r}} \frac{p_{\mathrm{F}} r^{2}}{e^{\tau}} d r .
$$

\section{NUMERICAL RESULTS}

Based on the discussion above we are now in position to estimate the photon flux and luminosity that can be produced by asymmetric dark stars. At first sight, what controls the photon production is the coefficient $\epsilon$. Clearly 
too small values of $\epsilon$ will lead to undetectable photon signal. As we mentioned earlier an induced photon mass will lead to an $\epsilon=\kappa m_{D}^{2} /\left(m_{D}^{2}-m_{A^{\prime}}^{2}\right)$. The kinetic mixing $\kappa$ is constrained experimentally [34, 35]. For heavy dark photon masses e.g. of the order of $m_{A^{\prime}}=1 \mathrm{GeV}$, $\kappa<10^{-5}$. Despite the fact that smaller dark photon masses are more constrained, they can provide a much larger luminosity in dark stars. The reason is twofold. Firstly the mass of the dark photon appears in the denominator of the flux equation (see e.g. eq. (32)) and therefore lighter dark photons produce larger luminosities. The second reason is also that light dark photons (for example lighter than the medium acquired photon mass $m_{D}$ ) saturate $\epsilon$ to $\kappa$ providing significant amount of mixing ${ }^{4}$. The value of $\epsilon$ or $\kappa$ can also affect the photon production in two different ways: generally large values of $\epsilon$ correspond to larger photon production since the latter scales as $\sim \epsilon^{2}$. However in principle, too strong $\epsilon$ can allow re-conversion of produced photons at the center of the star back to dark photons due to large cross section of a dark Compton process. If photons produced at the center are reabsorbed by the star, then the emitted photons will come from layers close to the surface leading to an overall reduction. This represents a major difference with respect to the emission from standard NS where the bulk of the star does not contribute due to the very short photon mean free path inside the core. As a result, the spectrum of photons produced via Bremsstrahlung inside dark stars is qualitatively different from that of NS. In the latter case, one should expect the usual black-body spectrum (sometimes deformed by the star's atmosphere) peaking roughly at a frequency close to the surface temperature of the star. On the contrary, the potential spectrum from dark stars is not solely produced from photons produced on the surface of the star, but it rather comes from the whole bulk redshifted appropriately depending on the depth produced. Therefore although the photon spectrum in dark stars is $\epsilon^{2}$ suppressed, this is to some extent counterbalanced by the fact that the whole volume of the star participates in the photon production. In addition, the fact that the produced photons are not in thermal equilibrium with the medium leads to a different shape in the spectrum compared to photon production from the surface of a NS where the photons are always in thermal equilibrium with the nuclear matter and therefore the spectrum is that of black-body. The photon spectrum from dark stars in shown Fig. 4, where we plot the flux of received photons per energy, namely $d^{3} N /\left(d S d t_{\infty} d \omega_{\infty}\right)$ in eq. (24). For energies smaller than $\sim T$, the spectrum is determined by the characteristic $\omega_{\infty}^{-1}$ of the Bremsstrahlung rate, while the rate drops faster above $\sim T$ due to a thermal exponential suppression. Overall, we have tried different parameter values

\footnotetext{
${ }^{4}$ In fine tuned situations where $m_{D} \simeq m_{A^{\prime}} \epsilon$ can be become in principle much larger than $\kappa$. We will not examine such fine tuned cases here.
}

for heavy dark photons within the allowed range of $\kappa$ and despite the fact that the star is not opaque to the produced photons i.e., the mean free path of the photons is much larger than the size of the star and therefore all photons make it out from the star, the produced photon spectrum is too small to be detected within current observational capabilities. As shown in Fig. 4 the situation changes once we consider light dark photons, because as we mentioned not only they lead to larger mixing with the ordinary photons, but they also give an overall higher amplitude.

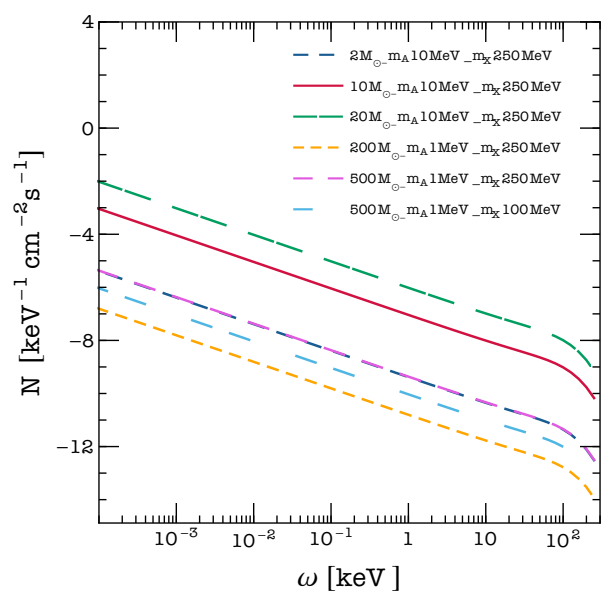

FIG. 4. Flux of received photons as a function of the energy for different dark stars at a distance $d=1 \mathrm{kpc}$, with temperature of $T=5 \times 10^{8} \mathrm{~K}$. Coloured curves refer to different values of the dark particle, dark photon and stellar masses. For $m_{A}^{\prime}=10 \mathrm{MeV}\left(m_{A}^{\prime}=1 \mathrm{MeV}\right)$ we fix $\kappa=10^{-4}\left(\kappa=10^{-8}\right)$.

Figures 5 show the photon energy flux (25) produced by a large variety of dark stars with various masses and EoS. We consider sources at a prototype distance of $d=1 \mathrm{kpc}$. However, since $\mathcal{F}$ is proportional to $1 / d^{2}$, these results can be immediately rescaled to any location. In Fig. 5 in particular we draw $\mathcal{F}$ as a function of the normalised temperature $T_{7}=T / 10^{7} \mathrm{~K}$ for dark stars with $m_{A}^{\prime}=10 \mathrm{MeV}$ and $m_{A}^{\prime}=1 \mathrm{MeV}$. We use two prescriptions for $\kappa$, choosing $\kappa=10^{-4}$ and $\kappa=10^{-8}$ for the heavy and the light dark photon, respectively. The latter plays a key role in determining the amplitude of the flux, which is overall favoured by small values of $m_{A}^{\prime}$. Note that $\mathcal{F}$ in eq. (32) is proportional to $T^{4}$, so these results can be extrapolated to any temperature by the appropriate rescaling. For $T \lesssim 5 \times 10^{8} \mathrm{~K}$ all the stellar models considered lead to values of $\mathcal{F}$ smaller than $1 \mathrm{eV} \mathrm{cm} \mathrm{cm}^{-2} \mathrm{~s}^{-1}$. The Bremsstrahlung fluxes grow with the temperature, and can be as high as $\mathcal{F} \sim 10^{2} \mathrm{eV} \mathrm{cm}^{-2} \mathrm{~s}^{-1}$ for massive stellar configurations with $M \gtrsim 50 M_{\odot}$ and $T=10^{9} \mathrm{~K}$. Although the flux in eq. (32) depends directly on the mass of the $\mathrm{DM}$ particles as $\mathcal{F} \sim m_{\mathrm{X}}^{2}$, it also depends indirectly on $m_{\mathrm{X}}$ because the latter affects the stellar density profiles, namely the stiffness of the fermion star. Indeed for fixed particle parameters (i.e., DM and dark photon mass, $\kappa$ and coupling $\alpha_{X}$ ), more 
massive stars have higher luminosities.

At this point we can make a comparison between the luminosity of an asymmetric dark star with that of a NS. Assuming a thermally cooling weakly magnetised NS, the flux produced at the surface is proportional to the stellar surface temperature, $\mathcal{F}_{\mathrm{NS}}=\sigma_{\mathrm{B}} T_{\mathrm{sur}}^{4} R^{2} / d^{2}$, where $\sigma_{\mathrm{B}}$ is the Stefan-Boltzmann constant and $T_{\text {sur }}$ the surface temperature of the NS. A typical NS of $1.5 M_{\odot}$ and $R \sim 11 \mathrm{~km}$ at $d=1 \mathrm{kpc}$, with a temperature within the range $\left[10^{7}, 10^{9}\right] \mathrm{K}$, provides a flux of $\mathcal{F}_{\mathrm{NS}} \in\left[10^{5}, 10^{13}\right] \mathrm{eV}$ $\mathrm{cm}^{-2} \mathrm{~s}^{-1}$ on Earth. As we already mentioned the luminosity of a dark star scales also as $T^{4}$ (see eq. (32)). Therefore for given DM parameters and mass of the dark star, its photon energy flux will always be a specific fraction of that of a typical NS with the same temperature located at the same distance from the Earth. On the other hand, larger values of $\kappa$, or smaller distances may change this picture. Let's take for example the two pulsars J0437-4715 and J0108-1431, which are about 140pc and 130pc away from the Earth, and have surfaces temperatures of $\sim 10^{5} \mathrm{~K}[36,37]$. All the dark stars shown in the left panel of Fig. 5 with $M \gtrsim 50 M_{\odot}$ at the same distance, would produce a flux higher than J0437-4715 and J0108-1431, provided that their temperature is larger than $T \sim 10^{7} \mathrm{~K}$.

A couple of comments are in order here. NS evacuate energy from the bulk via neutrino emission. In fact the modified Urca process is the main mechanism of NS cooling that dominates over surface photon emission in temperatures above $\sim 10^{8} \mathrm{~K}$. A process that would be somewhat analogous to our dark Bremsstrahlung is the neutrino Bremsstrahlung emission inside a NS studied in [38]. However this process scales parametrically differently with temperature from our case, since they are two thermal particles produced (a pair of neutrinoantineutrino) instead of one (the photon) in our case. A second point is that the dark star luminosity (as it can be seen in Fig. 5) can be significantly higher if the value of $\alpha_{X}$ is larger. For a given DM and dark photon mass, the value of $\alpha_{X}$ has an upper bound due to constraints on DM self-interactions from the bullet cluster. However, this constraint does not apply if $X$ does not account for the whole DM abundance but only a fraction of it.

\section{CONCLUSION}

Asymmetric DM is an attractive alternative to the thermally produced WIMP paradigm. Due to an inherited asymmetry between particles and antiparticles, DM annihilations are absent once the population of antiparticles is depleted. Therefore in case such a DM candidate possesses an effective mechanism of evacuating energy, it has been recently demonstrated [17] that such a DM component could collapse and form compact objects. In fact this is also desired in the view of the supermassive black holes which seem in need of seeds other than typical stellar remnants.

Up to now, it was considered that asymmetric dark stars could be detected only via gravitational wave signals. In fact in previous work, we had investigated and computed the tidal deformabilities of such stars and the prospects of their discovery via gravitiational waves [24]. In this paper we demonstrate that in some cases dark stars might also be detected by direct observation of a photon spectrum produced inside such a star via a dark Bremsstrahlung provided that dark photons mix kinetically with ordinary photons. We calculated the explicit form of the photon flux in terms of the stellar and EoS parameters. We numerically computed the emitted flux for a variety of model parameters. Obviously the overall process is strongly affected by the distance between the Earth and the dark star and by the kinetic mixing. We found that the numbers of emitted photons is enhanced for heavier/larger dark stars. Depending on the stellar temperature, the Bremsstrahlung flux can be as

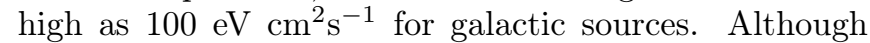
this process is in general smaller than the energy emitted by standard NS due to black-body radiation, the dark photon spectrum features a spectrum morphology, which is completely different from a thermal black-body component, thus providing a distinct discovery signature for dark stars. Finally it will be interesting to determine the rate of cooling for such asymmetric dark stars which will be dominated by emission (via dark Bremsstrahlung) of dark photons. In case where DM is admixed with baryonic matter and electrons, it will also be interesting to study how these particles affect the overall cooling and luminosity of the dark star. In addition, compact asymmetric dark stars can be composed not only of fermionic $\mathrm{DM}$ as in the case studied here but also of bosonic DM (e.g. studied in [12]). In this case the Bremsstrahlung mechanism can work in a similar way as in our current study. However, the emissivity will be different due to the fact that in the bosonic case there is no Fermi surface. In our present study only DM particles sitting within $\sim T$ from the Fermi surface can interact and produce photons. This Pauli blocking is not present in the case of bosonic stars and therefore the amount of produced photons could potentially be larger than the one studied here. Therefore the calculation is completely different. We will address all the above in future work.

Acknowledgements. - We would like to thank M. Tytgat for useful discussions. A.M. acknowledge support from the Amaldi Research Center funded by the MIUR program "Dipartimento di Eccellenza" (CUP: B81I18001170001). CK is partially funded by the Danish National Research Foundation, grant number DNRF90, and by the Danish Council for Independent Research, grant number DFF 4181-00055. A.M. and K.K would like to acknowledge networking support by the COST Action CA16214. 

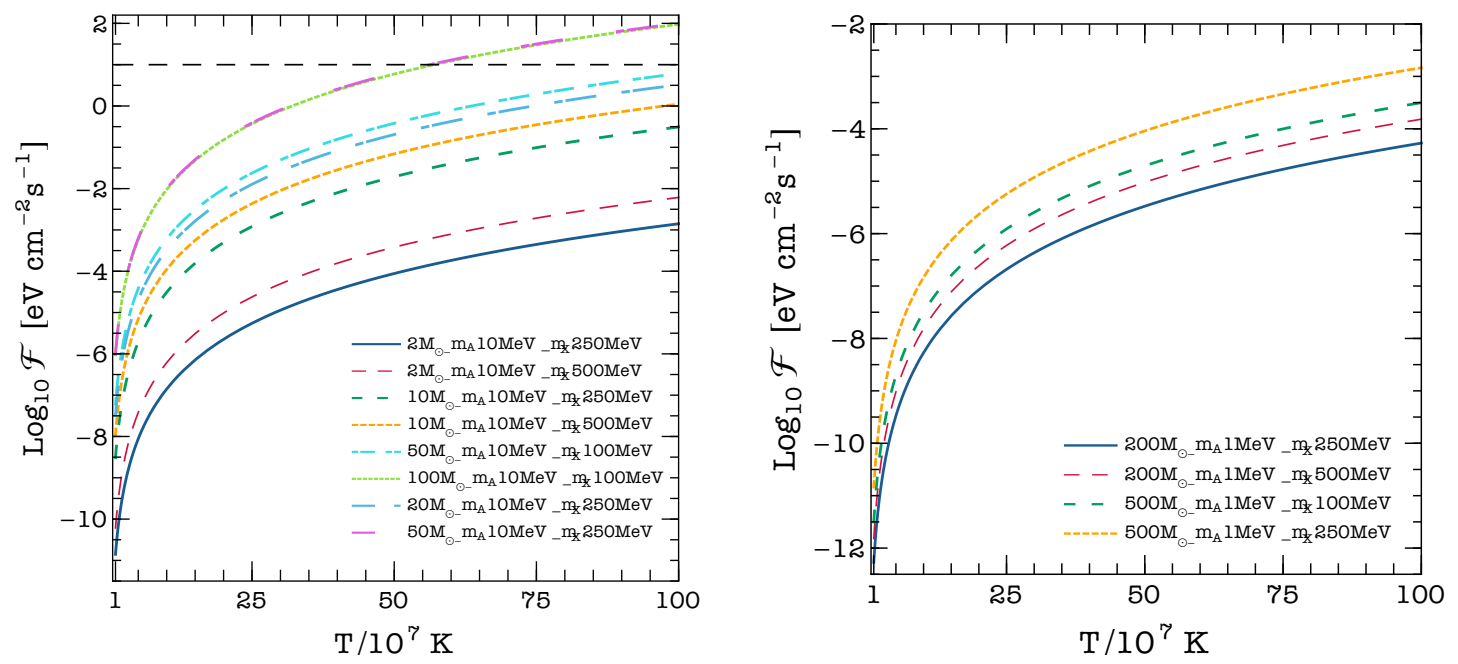

FIG. 5. Bremsstrahlung photon energy flux produced by dark stars located at a distance $d=1 \mathrm{kpc}$ away from the detector with $m_{\mathrm{X}}=(100,250,500) \mathrm{MeV}$ and $m_{A^{\prime}}=10 \mathrm{MeV}$ (left panel), $m_{A^{\prime}}=1 \mathrm{MeV}$ (right panel), as a function of the star's temperature. We consider different values of the stellar mass, fixing $\kappa=10^{-4}$ for $m_{A^{\prime}}=10 \mathrm{MeV}, \kappa=10^{-8} m_{A^{\prime}}=1 \mathrm{MeV}$, and $\alpha_{\mathrm{X}}=10^{-1}$. The dashed horizontal line corresponds to $\mathcal{F}=10 \mathrm{eV} \mathrm{cm}^{-2} \mathrm{~s}^{-1}$.

[1] D. Clowe, M. Bradac, A. H. Gonzalez, M. Markevitch, S. W. Randall, C. Jones, and D. Zaritsky, Astrophys. J. 648, L109 (2006), arXiv:astro-ph/0608407 [astro-ph].

[2] P. A. R. Ade et al. (Planck), Astron. Astrophys. 594, A13 (2016), arXiv:1502.01589 [astro-ph.CO].

[3] B. Moore, Nature 370, 629 (1994).

[4] R. A. Flores and J. R. Primack, Astrophys. J. 427, L1 (1994), arXiv:astro-ph/9402004 [astro-ph].

[5] J. F. Navarro, C. S. Frenk, and S. D. M. White, Astrophys. J. 490, 493 (1997), arXiv:astro-ph/9611107 [astro$\mathrm{ph}]$.

[6] M. Boylan-Kolchin, J. S. Bullock, and M. Kaplinghat, Mon. Not. Roy. Astron. Soc. 415, L40 (2011), arXiv:1103.0007 [astro-ph.CO].

[7] K. A. Oman et al., Mon. Not. Roy. Astron. Soc. 452, 3650 (2015), arXiv:1504.01437 [astro-ph.GA].

[8] R. Kuzio de Naray, G. D. Martinez, J. S. Bullock, and M. Kaplinghat, Astrophys. J. 710, L161 (2010), arXiv:0912.3518 [astro-ph.CO].

[9] S. Tulin and H.-B. Yu, Phys. Rept. 730, 1 (2018), arXiv:1705.02358 [hep-ph].

[10] J. Pollack, D. N. Spergel, and P. J. Steinhardt, Astrophys. J. 804, 131 (2015), arXiv:1501.00017 [astroph.CO].

[11] C. Kouvaris and N. G. Nielsen, Phys. Rev. D92, 063526 (2015), arXiv:1507.00959 [hep-ph].

[12] J. Eby, C. Kouvaris, N. G. Nielsen, and L. C. R. Wijewardhana, JHEP 02, 028 (2016), arXiv:1511.04474 [hepph].

[13] L. Tolos and J. Schaffner-Bielich, Phys. Rev. D92, 123002 (2015), arXiv:1507.08197 [astro-ph.HE].

[14] M. Deliyergiyev, A. Del Popolo, L. Tolos, M. Le Delliou, X. Lee, and F. Burgio, Phys. Rev. D99, 063015 (2019), arXiv:1903.01183 [gr-qc].

[15] C. Kouvaris, P. Tinyakov, and M. H. G. Tytgat, Phys. Rev. Lett. 121, 221102 (2018), arXiv:1804.06740 [astro-
ph.HE].

[16] M. I. Gresham and K. M. Zurek, (2018), arXiv:1809.08254 [astro-ph.CO].

[17] J. H. Chang, D. Egana-Ugrinovic, R. Essig, and C. Kouvaris, JCAP 1903, 036 (2019), arXiv:1812.07000 [hepph].

[18] V. Cardoso, E. Franzin, A. Maselli, P. Pani, and G. Raposo, Phys. Rev. D95, 084014 (2017), [Addendum: Phys. Rev.D95,no.8,089901(2017)], arXiv:1701.01116 [gr-qc].

[19] A. Maselli, P. Pani, V. Cardoso, T. Abdelsalhin, L. Gualtieri, and V. Ferrari, Phys. Rev. Lett. 120, 081101 (2018), arXiv:1703.10612 [gr-qc].

[20] V. Cardoso and P. Pani, Nat. Astron. 1, 586 (2017), arXiv:1709.01525 [gr-qc].

[21] L. Barack et al., (2018), arXiv:1806.05195 [gr-qc].

[22] V. Cardoso and P. Pani, (2019), arXiv:1904.05363 [grqc].

[23] P. Pani and A. Maselli, (2019), arXiv:1905.03947 [gr-qc].

[24] A. Maselli, P. Pnigouras, N. G. Nielsen, C. Kouvaris, and K. D. Kokkotas, Phys. Rev. D96, 023005 (2017), arXiv:1704.07286 [astro-ph.HE].

[25] H. An, M. Pospelov, and J. Pradler, Phys. Lett. B 725, 190 (2013), arXiv:1302.3884 [hep-ph].

[26] J. Redondo and G. Raffelt, JCAP 08, 034 (2013), arXiv:1305.2920 [hep-ph].

[27] P. Arias, D. Cadamuro, M. Goodsell, J. Jaeckel, J. Redondo, and A. Ringwald, JCAP 06, 013 (2012), arXiv:1201.5902 [hep-ph].

[28] A. Ayala, I. Lopes, A. García Hernández, J. C. Suárez, and I. n. Muñoz Elorza, Mon. Not. Roy. Astron. Soc. 491, 409 (2020), arXiv:1910.11827 [astro-ph.SR].

[29] X. Chu, J.-L. Kuo, J. Pradler, and L. Semmelrock, Phys. Rev. D 100, 083002 (2019), arXiv:1908.00553 [hep-ph].

[30] W. DeRocco, P. W. Graham, D. Kasen, G. MarquesTavares, and S. Rajendran, JHEP 02, 171 (2019), arXiv:1901.08596 [hep-ph]. 
[31] S. L. Shapiro and S. A. Teukolsky, Research supported by the National Science Foundation. New York, WileyInterscience, 1983, 663 p. (1983).

[32] C. Kouvaris and J. Pradler, Phys. Rev. Lett. 118, 031803 (2017), arXiv:1607.01789 [hep-ph].

[33] V. Berestetskii, L. Pitaevskii, and E. Lifshitz, Quantum Electrodynamics, v. 4 (Elsevier Science, 2012).

[34] J. Jaeckel, Frascati Phys. Ser. 56, 172 (2012), arXiv:1303.1821 [hep-ph].

[35] M. Bauer, P. Foldenauer, and J. Jaeckel, JHEP 07, 094
(2018), arXiv:1803.05466 [hep-ph].

[36] O. Kargaltsev, G. G. Pavlov, and R. W. Romani, Astrophys. J. 602, 327 (2004), arXiv:astro-ph/0310854 [astro$\mathrm{ph}]$.

[37] R. P. Mignani, G. G. Pavlov, and O. Kargaltsev, Astron. Astrophys. 488, 1027 (2008), arXiv:0805.2586 [astro-ph].

[38] P. Jaikumar, C. Gale, and D. Page, Phys. Rev. D72, 123004 (2005), arXiv:hep-ph/0508245 [hep-ph]. 